\title{
Chandra and VLA Observations of Supermassive Black Hole Outbursts in M87
}

\author{
William Forman ${ }^{1}$, C. Jones ${ }^{1}$, and Eugene Churazov ${ }^{2}$ \\ ${ }^{1}$ Smithsonian Astrophysical Observatory \\ 60 Garden St., Cambridge, MA USA \\ email: wrf@cfa.harvard.edu, cjones@cfa.harvard.edu \\ ${ }^{2}$ Max Planck Institute for Astrophysics, Garching Germany \\ Institute for Space Research, Moscow, Russia \\ email: churazov@MPA-Garching.MPG.DE
}

\begin{abstract}
We discuss the effects of supermassive black hole (SMBH) outbursts on the hot atmospheres surrounding the central massive galaxies in groups and clusters, as observed with $\mathrm{X}$-ray and radio observations. We focus on a detailed study of the supermassive black hole in M87 at the center of the Virgo cluster using Chandra and VLA observations. We summarize the outburst history and describe the clearly observed energy input from buoyant bubbles of relativistic plasma produced by the central $\mathrm{SMBH}$, uplifted filaments of X-ray emitting gas, and the Mach 1.2 shock together balance the energy lost as gas radiatively cools.
\end{abstract}

Keywords. galaxies: clusters: individual (M87), galaxies: active, galaxies: evolution

\section{Introduction}

Outbursts from supermassive black holes have become an important ingredient of galaxy evolution models (e.g., Croton et al. 2006, Bower et al. 2006, Guo et al. 2011). $\mathrm{X}$-ray observations of gas rich systems from clusters to groups to galaxies show that outbursts at the present epoch are very common and serve to replenish the energy lost from radiative cooling in hot gas rich, massive galaxies. M87, the dominant central galaxy of the Virgo cluster, is a classic example of a cooling flow cluster (e.g., Fabian 1994) and is the nearest example of this class. In the absence of any energy input, the inferred cooling rate around M87 implies a cooling flow of $\sim 10 \mathrm{M}_{\odot} \mathrm{yr}^{-1}$ (Stewart et al. 1984). M87 is the second brightest extragalactic X-ray source (Forman et al. 1978) and is 50 times more X-ray luminous than NGC4472, the optically brightest galaxy in Virgo. M87 hosts a $6.6 \times 10^{9} \mathrm{M}_{\odot} \quad \mathrm{SMBH}$ (Gebhardt et al. 2011) and a well-studied jet, detected from the radio to the X-ray regime (Marshall et al. 2002), that is inflating a plasma filled cocoon. With a massive $\mathrm{SMBH}$, an associated jet, and an X-ray luminous atmosphere, M87 is the ideal system for detailed studies of the feedback process between a SMBH and its surrounding gaseous corona (see Churazov et al. 2001, Forman et al. 2005, Forman et al. 2007, Million et al. 2010).

\section{M87's Environment - Merger Driven Gas Sloshing}

The Virgo cluster core, dominated by M87, is a complex environment and is still undergoing mergers. Fig. 1 (left panel) shows the wide field ROSAT image of the cluster core including the "cold front" around M87 and the dramatic merger of M86 with its more than $150 \mathrm{kpc}$ long ram pressure stripped tail (Randall et al. 2008). The mergers in Virgo have perturbed the core and are driving "gas sloshing" (Markevitch \& Vikhlinin 2007 

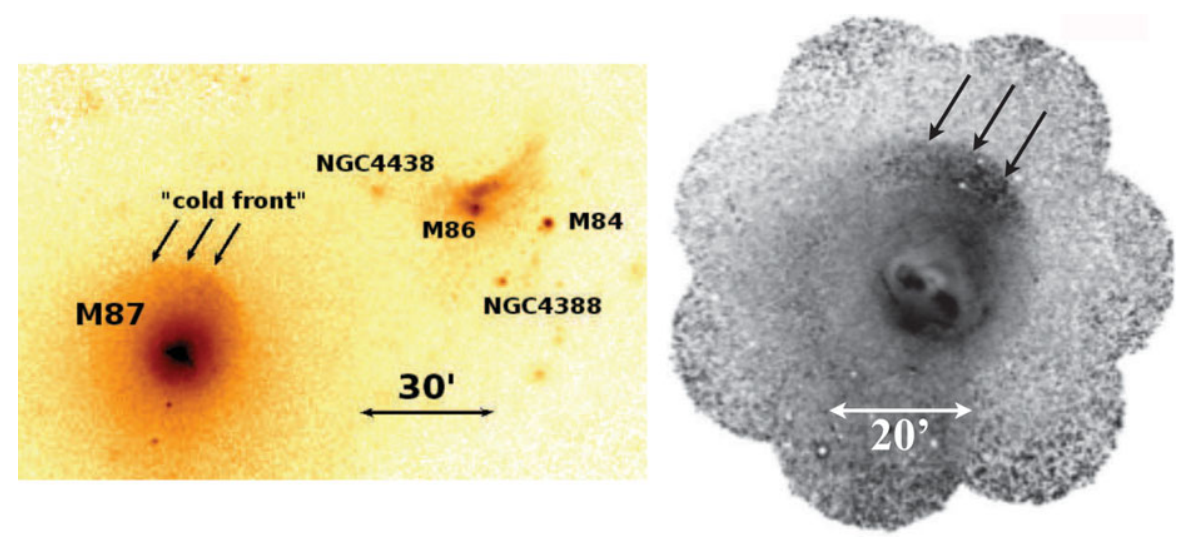

Figure 1. (left)ROSAT view of the X-ray emission surrounding M87. In addition to the very bright emission from M87, we see M86 (NGC4406) and its ram pressure stripped tail, as well as emission from other bright galaxies in the Virgo core, including M84 (NGC4374), NGC4438, and NGC4438. (right) The XMM-Newton observatins of M87 with an average surface brightness profile subtracted shows the sloshing cold front (dark arrows), as well as the cool gas "arms" (extending $5^{\prime}$ to the east and southwest).

for a review; see Simionescu et al. 2010 for details of M87). Note that merger-driven gas sloshing is commonly seen in clusters with gas density peaks (14 clusters in a sample of 18 show similar features; Markevitch et al. 2003; Johnson 2011). Fig. 1 (right panel) shows the XMM-Newton image of the core region with an average radial profile subtracted to enhance faint features. The northern X-ray edge, a contact discontinuity (see Markevitch \& Vikhlinin 2007 for a detailed review of cold fronts) is clearly seen (black arrows) as are the X-ray filamentary structures in the very central region extending to the east and southwest (see Fig. 2 (left panel) for the Chandra high resolution view of the filaments).

\section{Shocks and Filaments in M87}

The VLA and Chandra observations of M87 show a sequence of outbursts in M87 that are chronicled in Fig. 2 and can be summarized as:

- The outer radio "pancakes" (filamentary circular regions in Fig. 2 right panel; adapted from Owen, Eilek \& Kassim 2000). Fig. 1 in Churazov et al. (2001), described these as buoyant bubbles flattened into "pancakes" as they rose in M87's atmosphere. Owen et al. (2000) estimate the ages of these largest lobes as $100-150 \times 10^{6} \mathrm{yr}$.

- "mushroom cloud" with stem and torus (dark structure in Fig. 2, right panel) extending to the east from the nucleus with an age of a few tens of Myr (the buoyancy time). A radio structure also extends to the southwest, but has been disrupted. These two radio plasma arms are closely related to the X-ray filamentary arms (Fig. 2, left panel) of cool gas that has been uplifted by the buoyant radio-emitting bubbles.

- $13 \mathrm{kpc}$ shock, a nearly complete azimuthal ring (Fig. 2, left center panel, the hard X-ray image of M87) at a radial distance of $13 \mathrm{kpc}\left(2.8^{\prime}\right)$ from the center of M87, driven by an outburst $\sim 12 \times 10^{6}$ yr old, and its driving piston, the central radio lobes.

- a possible weaker shock at about $5 \mathrm{kpc}$ (about $5 \times 10^{6} \mathrm{yr}$ old first suggested as a weak shock in Forman et al. 2007; see also Million et al. 2010).

- the present outburst with its multi-wavelength jet, flaring knots, and variable gammaray emission (Hines et al. 1989; Owen et al. 2000; Marshall et al. 2002; Harris et al. 2006; Shi et al. 2007; Acciari et al. 2010). 

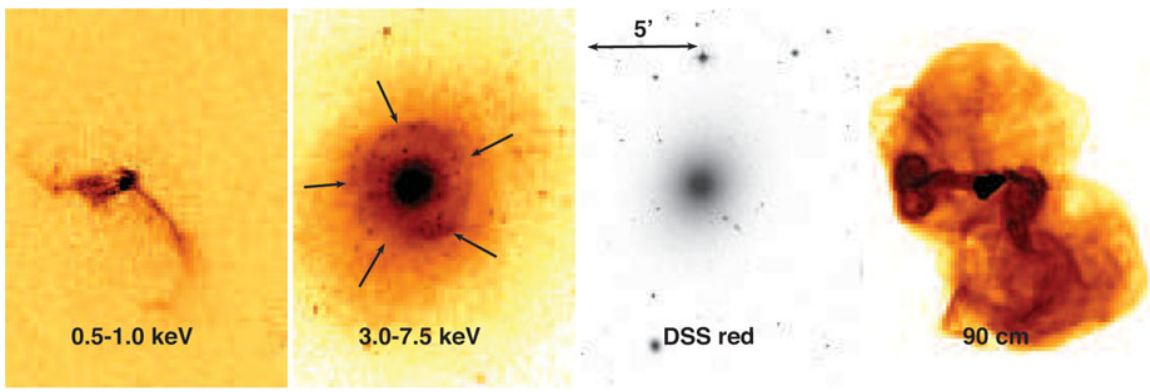

Figure 2. Images of M87 from soft and hard X-ray to optical and radio at the same angular scale showing the variety of outbursts. (left) The soft $(0.5-1.0 \mathrm{keV})$ Chandra image showing the thin filamentary arms, uplifted by the buoyant radio bubbles; (left-center) The hard (3.0-7.5 keV) Chandra image showing the main shock. The hard band image is a "pressure map" (integral of pressure squared projected on the sky) and directly shows the $13 \mathrm{kpc}$ shock (dark arrows); (center-right) The DSS (red) image of M87 showing the galaxy scale compared to the X-ray and radio; (right) The $90 \mathrm{~cm}$ VLA map of M87 (Owen et al. 2000) which shows the large relativistic plasma bubbles as well as the "mushroom cloud" feature buoyantly rising to the east and the disrupted arm extending to the southwest. The radio bright nuclear region, the piston that initiated the $13 \mathrm{kpc}$ outburst, is clearly visible in the $90 \mathrm{~cm}$ radio image.

Hence, M87 shows a rich sequence of outbursts. We discuss the $13 \mathrm{kpc}$ shock and its associated central "piston" which present the opportunity, through a simple and robust model, to derive the characteristics of a SMBH outburst and the energy partition between the shock and the central buoyant radio lobes.

Fig. 3 (left) shows the azimuthally averaged (northern sector of width $80^{\circ}$ ) surface brightness profiles in the 1.2-2.5 keV (upper curve) and 3.5-7.5 keV (lower curve) bands (see Forman et al. 2007 for details). The profiles show a sharp edge at $\sim 0.6^{\prime}$ (most distinctly seen in the hard band as a decrease between the fourth and fifth data points), a moderate flattening of the profile at about $1^{\prime}$, and a strong excess at $2-3^{\prime}$. This strong excess is the $13 \mathrm{kpc}$ shock. To derive quantitative estimates of the shock jump conditions, we deprojected surface brightness profiles into emissivity profiles (Fig. 3 central panel). The deprojection shows the very pronounced $13 \mathrm{kpc}$ shock at $2^{\prime}-3^{\prime}$.

The 1.2-2.5 keV energy band is nearly a direct measure of the gas density. The soft and hard bands combine to yield the temperature profile. The derived values of the gas density and temperature jumps are $\rho_{\text {shock }} / \rho_{0}=1.33 \pm 0.02$ and $T_{\text {shock }} / T_{0}=1.18 \pm 0.03$ (see Forman et al. 2007 for details). These values, combined with the Rankine-Hugoniot shock jump conditions, yield independent, consistent values of the Mach number $M \sim 1.2$.

For the $13 \mathrm{kpc}$ shock and its driving outburst, we describe a simple (one dimensional model) that yields the energy balance between shock and "bubble" energy. Constraints on the model include 1) the density and temperature jumps across the shock which yield the present epoch shock Mach number and 2) the size of the driving piston as seen in the radio-filled central cavity. The Mach number yields the outburst energy (almost directly) of about $5 \times 10^{57}$ ergs. The combined constraints give an estimate of the outburst duration of about $2 \mathrm{Myr}$. The present radius of the shock, in the context of the model, gives an age of about $12 \mathrm{Myr}$. The model shows that over the lifetime of the outburst, the energy is partitioned with about half of the energy in the enthalpy of the central piston (cavity/bubble), about a quarter possibly going into shock heating of the gas, and the remaining part is carried to the outskirts of the cluster as the shock weakens to a sound wave. Averaged over the age of the outburst (to the time of today's present outburst), the outburst power matches the radiative cooling of the gas in the cluster core. 

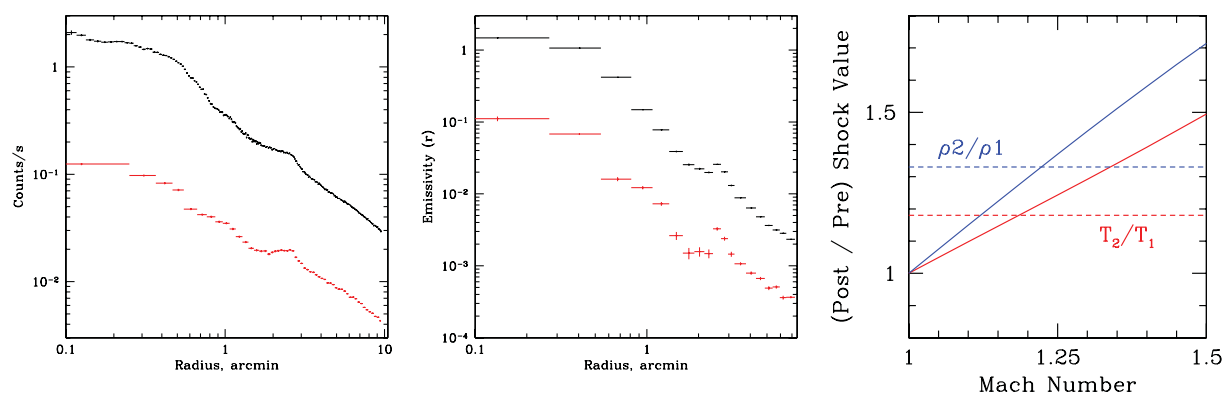

Figure 3. The radial profiles and Mach number of the $13 \mathrm{kpc}$ shock in M87. (left panel) The azimuthally averaged (sector of width $80^{\circ}$ centered on north) surface brightness profiles in the 1.2-2.5 keV (upper curve) and 3.5-7.5 keV (lower curve) bands. The $13 \mathrm{kpc}$ shock is seen as the excess at $2-3^{\prime}$. (central panel) The deprojected emissivity profiles, derived from the curves in the left panel. The deprojection shows the very pronounced $13 \mathrm{kpc}$ shock at $2^{\prime}-3^{\prime}$. (right panel) The ratio of the pre-shock and post-shock density (blue solid line) and temperature (red solid line) as a function of shock Mach number as defined by the Rankine-Hugoniot shock jump conditions. The measured ratios of pre/post shock density and temperature are shown as dashed horizontal lines (blue and red for density and temperature, respectively). The intersections of the predicted and measured functions give consistent Mach numbers of $\sim 1.2$.

\section{Conclusions}

M87, the nearest active galaxy at the center of a gaseous cluster atmosphere shows multiple outbursts in its radio and X-ray images. We see evidence for both shocks and for buoyant relativistic plasma bubbles providing energy to the cluster ICM. A simple model gives details of the partition of energy with roughly $50 \%$ in bubbles which will be returned to the hot gas and $50 \%$ in the $13 \mathrm{kpc}$ shock of which half is used to heat the gas and half is carried away by the shock as it becomes a weak sound wave.

\section{References}

Acciari et al. 2010, ApJ, 716819

Bower, R. et al. 1993, MNRAS, 370, 645

Churazov, E., Brueggen, M. Kaiser, C., Boehringer, H., \& Forman, W. 2001, ApJ, 554, 261

Croton, D. et al. 1995, MNRAS, 365, 11

Forman, W. et al. 1978 1978, ApJS, 38, 357

Gebhardt, K. et al. 2011, ApJ, 729, 119

Guo, Q. et al. 2011, MNRAS, 413, 101

Harris, D. et al. 2006, ApJ, 640, 211

Hines, D., Eilek, J., \& Owen, F. 1989, ApJ, 347, 713

Johnson, R. 2011, Ph.D. Thesis, Dartmouth College, Publication number: AAT 3477679

Markevitch, M. \& Vikhlinin, A. 2007, Phy. Rev, 443, 1

Markevitch, M., Vikhlinin, A., \& Forman, W. 2003, ASPC 301, 37

Marshall, H. et al. 2002, ApJ, 564, 683

Million, E. et al. 2010, MNRAS, 407, 2046

Owen, F. Eilek, J., \& Kassim, N. 2000, ApJ, 543, 611

Randall, S. et al. 2008, ApJ, 688, 208

Shi, Y., Rieke, G., Hines, D., Gordon, K., \& Egami, E. 2007, ApJ 655, 781

Simionescu, A. et al. 2010, MNRAS, 405, 91

Stewart, G., Canizares, C., Fabian, A., \& Nulsen, P. 1984, ApJ, 278, 536 